\title{
Low Grade Appendiceal Mucinous Neoplasm-A Rare Case Presentation
}

\author{
Suhailur Rahman, Binjul Juneja, Feroz Alam, and Kafil Akhtar* \\ Department of Pathology, Jawaharlal Nehru Medical College, Faculty of Medicine, Aligarh Muslim University, India
}

${ }^{*}$ Corresponding author: Kafil Akhtar, Department of Pathology, Jawaharlal Nehru Medical College, Aligarh Muslim University, Aligarh, India, E-mail: drkafilakhtar@gmail.com

Received: 29 Oct, 2019 | Accepted: 06 Jan, 2020 | Published: 10 Jan, 2020

Citation: Rahman S, Juneja B, Alam F, Akhtar K (2020) Low Grade Appendiceal Mucinous Neoplasm-A Rare Case Presentation. Clin Res Open Access 6(1): dx.doi.org/10.16966/2469-6714.152

Copyright: (C 2020 Rahman S, et al. This is an open-access article distributed under the terms of the Creative Commons Attribution License, which permits unrestricted use, distribution, and reproduction in any medium, provided the original author and source are credited.

\begin{abstract}
The most common epithelial tumor affecting the cecal appendix is mucinous neoplasm. Malignant mucinous neoplasms of the appendix are rare entities, often asymptomatic. Low-Grade Appendiceal Mucinous Neoplasms (LAMN) are most often clinically silent. One of the rare causes of acute abdomen is appendiceal mucinous neoplasm, representing only $0.2-0.7 \%$ of all appendectomy specimens. These tumors are discovered incidentally either during a survey or at the time of surgery for other causes. Mucinous neoplasms of the appendix carry a complex diverse group of neoplasms ranging from simple mucoceles to complex pseudomyxoma peritonei. We present a rare case report of a 27 year old female, who was admitted to the surgical emergency with severe pain and tenderness in the right abdomen for 3 days. Emergency laparotomy with appendectomy was performed and based on the histopathological findings; a diagnosis of low grade appendiceal mucinous neoplasm (LAMN) was given. Our patient has recovered completely postoperatively and is well after 6 months of follow up period.
\end{abstract}

Keywords: Low grade appendiceal neoplasm; Histopathology; Mucinous neoplasms; Ultrasonography

\section{Introduction}

Appendiceal mucinous neoplasms are tumors with neoplastic adenomatous growth of the appendix. Rokitansky first described this lesion in 1842 as the abnormal deposition of mucinous material in the appendiceal lumen or mucosal hyperplasia causing its dilatation. Approximately, $63-84 \%$ of the neoplastic cases are benign hyperplasias [1]. Appendiceal mucoceles were initially subdivided into four groups: simple mucocele, mucosal hyperplasia, mucinous cystadenoma and mucinous cystadenocarcinoma [2].

The Peritoneal Surface Oncology Group International (PSOGI) have recommended using the 2016 Modified Delphi Consensus Protocol to classify non-carcinoid epithelial appendiceal tumors into eight histomorphological architectural groups: adenoma, serrated polyp, low-grade appendiceal mucinous neoplasm (LAMN), high-grade appendiceal mucinous neoplasm (HAMN), mucinous adenocarcinoma (well/moderately/poorly differentiated), signet ring cell low-differentiated (mucinous) adenocarcinoma, signet-ring cell (mucinous) adenocarcinoma, and adenocarcinoma [3].

On the other hand, The American Joint Committee on Cancer (AJCC) $8^{\text {th }}$ edition clarifies LAMN staging to include prognostically relevant criteria, and describes a new $\mathrm{T}$ category specifically for LAMN, termed Tis (LAMN) [4]. Both the AJCC $8^{\text {th }}$ edition and the PSOGI consensus emphasize the importance of distinguishing between low-grade and high-grade intraperitoneal disease, and both advocate for three-tier grade assessment of appendiceal mucinous neoplasms, in which low-grade tumors are classified as G1, while high-grade tumors are classified as G2 or G3[4].
LAMN is most common in adult women. In $8.0 \%$ of the cases, it presents with clinical features of acute appendicitis [2]. LAMN may present as strangulation of intestine and appendiceal intussusceptions with features of abdominal mass with loss of weight and altered bowel habits [5]. Approximately 30.0\% patients of appendiceal mucinous neoplasms, irrespective of grade present with appendiceal perforation and mucin extravasation in the abdominal cavity, resulting in pseudomyxoma peritonei characterised by multiple mucinous implants [2].

We present a rare case report of a 27 year old female, who was admitted to the surgical emergency with severe pain and tenderness in the right abdomen for 3 days. Emergency laparotomy with appendectomy was performed and based on the histopathological findings; a diagnosis of low grade appendiceal mucinous neoplasm (LAMN) was given.

\section{Case Summary}

A 27 year old female presented to the surgical emergency with severe pain in the right abdomen for 3 days. The pain was nonradiating with no exacerbations or remissions and no aggravating or relieving factors. On examination, tenderness was felt in the right iliac fossa with no distension of abdomen or muscle guarding. Rest of the physical and systemic examinations was within normal limits. Routine blood investigations were normal. Ultrasonography of the abdomen showed $16 \mathrm{~mm}$ thick inflamed appendix with attached mesoappendix. Emergency exploratory laparotomy with appendectomy was performed. 
Macroscopically, the appendix was distended, $7.5 \times 1 \times 1 \mathrm{~cm}$ in size with slimy and congested outer surface and mucoid debris in the lumen. No foci of wall thinning or perforation were identified. Microscopically tissue sections showed papillary hyperplasia of mucosal epithelium, lined by mildly atypical cells with foci of stratification and mucin production (Figure 1). There was transmural inflammation along with few scattered PAS positive mucin containing tumor cells in the muscle layer (Figure 2). Serosa showed areas of well-defined mucin pool with few scattered cells with mild atypia. Based on the histopathological findings, a diagnosis of low grade appendiceal mucinous neoplasm (LAMN) was given. Our patient recovered completely postoperatively and is well after 6 months of follow up period.

\section{Discussion}

Low grade appendiceal mucinous neoplasms are most often clinically silent. They are an incidental finding and clinically present with features of acute pain in the right iliac fossa. Majority of them are

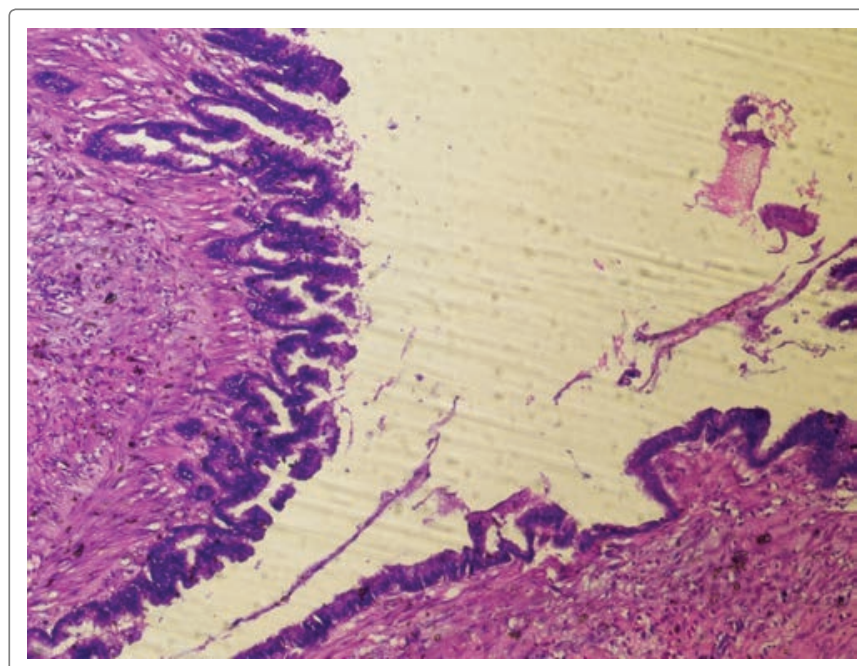

Figure 1: Microscopically tissue sections showed papillary hyperplasia of mucosal lining with mild atypical cells and focal mucin production, with well-defined mucin pool and few scattered mildly atypical appearing cells in the serosal fat. Hematoxylin and Eosin x10X.

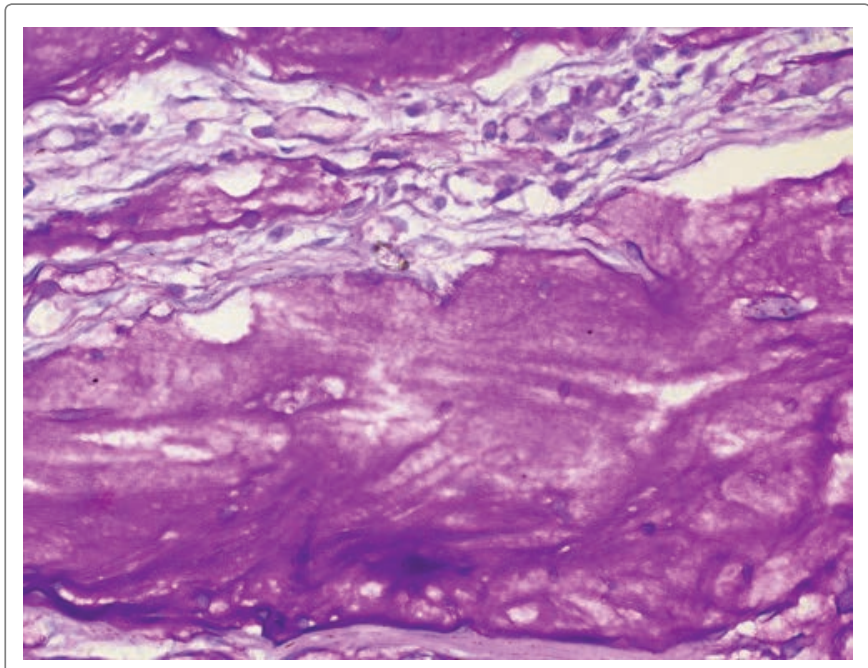

Figure 2: Tissue section shows transmural inflammation along with few scattered PAS positive mucin containing cells in the muscle layer. Periodic Acid Schiff x40X. diagnosed as acute appendicitis, with features of muscle guarding and mass on palpation. Benign appendiceal mucinous neoplasms are more common than the malignant variant, and accounts for $63-84 \%$ of the cases [6]. These lesions present as appendiceal enlargement with features of epithelial villous adenomatous changes with atypia [7].

LAMNs are low grade well-differentiated neoplasms but can spread beyond the appendix in a malignant fashion [8,9]. Low grade appendiceal neoplasm is defined as mucinous neoplasm with low grade cytological features such as loss of muscularis mucosa, fibrosis of submucosa, flattened or undulating epithelial growth, diverticulum or expansile like growth, dissection of a cellular mucin in the wall and mucin or malignant cells beyond the appendix (according to Peritoneal Surface Oncology Group International (PSOGI) classification) [3]. Mucinous adenocarcinoma is characterized by severe luminal distension as well as glandular invasion containing high-grade atypical cytology and extracellular mucin in more than $50.0 \%$ of the lesion [10].

Disseminated disease presents as pseudomyxoma peritonei, ovarian or abdominal masses in most patients. Pseudomyxoma peritonei is mucinous carcinoma with peritoneal cavity implants of neoplastic cells secreting mucin, leading to ascites. The ascitic fluid is characteristically gelatinous mucin in nature.

Ultrasound and Computed Tomography (CT) are worthwhile in the diagnosis of appendiceal mucinous neoplasms [11,12]. Ultrasonography has a sensitivity of $83.0 \%$ and specificity of $92.0 \%$ in detection of appendiceal mucinous neoplasms using threshold of $\geq 15 \mathrm{~mm}$. Ultrasound shows echogenic concentric layers ("onion skin") of lamellated mucin. CT scan is used for the confirmation of the diagnosis. Presence of curvilinear mural calcifications is suggestive of mucinous neoplasms in up to $50 \%$ of the patients [11]. Magnetic resonance imaging in mucinous neoplasms shows hyperintense tubular distention of the appendix [12].

Fine needle aspiration cytology is contraindicated in mucinous neoplasms to avoid spillage and perforation. Any fluid or mucus sampled should be examined cytologically. Operated lymph nodes must be evaluated in cases suspicious for malignancy. Immunohistochemical markers used to confirm the appendiceal tumor origin are CK20, CDX2 and SATB2 [13].

The differential diagnosis for LAMN includes mucinous adenocarcinoma of appendix, ruptured appendiceal diverticulum, serrated polyp and villous adenoma [14]. Appendiceal mucinous adenocarcinoma shows neoplastic tumor cell infiltration in the muscle layer with marked cytologic atypia and desmoplastic stromal reaction. Appendiceal diverticulum may rupture, with leakage of mucin and mimick low grade appendiceal mucinous neoplasm due to presence of mucin on the appendiceal serosa. Most cases of ruptured diverticula show reactive changes with maintained lamina propria as opposed to epithelial changes of neoplastic lesion. Serrated polyp presents as serrated lining epithelium and intact muscularis mucosae. Villous adenoma shows a focal tubulovillous architecture, with marked dysplastic features and retention of muscularis mucosae.

Appendectomy along with en-block appendicular mesenteric fat resection is the usual operative treatment of LAMNs [15]. No recurrence is reported within six year follow up in low grade mucinous neoplasms with a cellular mucin limited to the appendix. Five years of follow-up with six monthly CT scans along with tumor marker surveillance with CEA and CA19-9 should be done in LAMN's with perforation [13]. 


\section{Conclusions}

Low Grade Appendiceal Mucinous Neoplasms (LAMN) are most often clinically silent and these tumors are discovered incidentally either during a survey or at the time of surgery for other causes. So utmost care and caution should be exercised while examining any appendectomy specimen to not miss out on this rare diagnostic disease.

\section{References}

1. Carlos AG, Cleber SJ, Federico C, Giulia M, Rafaela CR, et al. (2018) Mucinous neoplasm of the appendix: management according to reviewed histologic spectral aspects. J peritoneum 3: 78-89.

2. Omari AH, Khammash MR, Qasaimeh GR, Shammari AK, Yaseen MK, et al. (2014) Acute appendicitis in the elderly: risk factors for perforation. World J Emerg Surg 9: 6-7.

3. Carr NJ, Cecil TD, Mohamed F, Sobin LH, Sugarbaker PH, et al. (2016) A consensus for classification and pathologic reporting of pseudomyxoma peritonei and Associated Appendiceal neoplasia: The results of the Peritoneal Surface Oncology Group International (PSOGI) modified Delphi process. Am J Surg Pathol 40: 14-26.

4. Overman MJ, Asare EA, Compton CC (2018) Appendix-carcinoma. In: Amin MB, Edge SB, Greene FL, Byrd DR, Brookland RK, et al. (eds) AJCC cancer staging manual. $8^{\text {th }}$ Edition, Springer International Publishing, Chicago, IL 237-250.

5. Yakan S, Caliskan C, Uguz A, Korkut MA, Coker A (2017) A retrospective study on mucocele of the appendix presented with acute abdomen or acute appendicitis. Hong Kong J Emerg Med 18: 144-149.

6. Miyakura $\mathrm{Y}$, Yokota S, Horie H, Togashi K, Lefor A, et al. (2012) Intestinal strangulation caused by a mucocele of the appendix: report of a case. Surg Today 42: 1215-1218.
7. Park JK, Kwon TH, Kim HK, Park JB, Kim K, et al. (2011) Adult intussusception caused by an appendiceal mucocele and reduced by colonoscopy. Clin Endosc 44: 133-136.

8. Kehagias I, Zygomala A, Markopoulos G, Papandreou T, Kraniotis $P$ (2016) Diagnosis and treatment of mucinous appendiceal neoplasm presented as acute appendicitis. Case Rep Oncol Med 2161952.

9. Carr N, Sobin L (2010) Tumors of the appendix. In: Bosman FT, Carneiro F, Hruban RH, Theise ND (eds) WHO Classification of tumours of the digestive system. $4^{\text {th }}$ Edition, World Health Organization classification of Tumours 3: 122-125.

10. Pai RK, Longacre TA (2005) Appendiceal mucinous tumors and pseudomyxoma peritonei: histologic features, diagnostic problems and proposed classification. Adv Anat Pathol 12: 291-311.

11. Tirumani SH, Fraser-Hill M, Auer R, Shabana W, Walsh $C$, et al. (2013) Mucinous neoplasms of the appendix: a current comprehensive clinicopathologic and imaging review. Cancer Imaging 13: 14-25.

12. Pickhardt PJ, Levy AD, Rohrmann CA, Kende Al (2003) Primary neoplasms of the appendix: radiologic spectrum of disease with pathologic correlation. Radiographics 23: 645-662.

13. Strickland S, Parra-Herran C (2016) Immunohistochemical characterization of appendiceal mucinous neoplasms and the value of special AT-rich sequence-binding protein 2 in their distinction from primary ovarian mucinous tumours. Histopathol 68: 977-987.

14. Misdraji J (2015) Mucinous epithelial neoplasms of the appendix and pseudomyxoma peritonei. Mod Pathol 28: S67-S79.

15. Sugarbaker PH (2009) Epithelial appendiceal neoplasms. Cancer J 15: 225-235. 